\title{
NCSU_SAS_SAM: Deep Encoding and Reconstruction for Normalization of Noisy Text
}

\author{
Samuel P. Leeman-Munk James C. Lester \\ Center for Educational Informatics \\ North Carolina State University \\ Raleigh, NC, USA \\ \{spleeman, lester\}@ncsu.edu
}

\author{
James A. Cox \\ Text Analytics R\&D \\ SAS Institute Inc. \\ Cary, NC, USA \\ james.cox@sas.com
}

\begin{abstract}
As a participant in the W-NUT Lexical Normalization for English Tweets challenge, we use deep learning to address the constrained task. Specifically, we use a combination of two augmented feed forward neural networks, a flagger that identifies words to be normalized and a normalizer, to take in a single token at a time and output a corrected version of that token. Despite avoiding off-the-shelf tools trained on external data and being an entirely context-free model, our system still achieved an F1-score of $81.49 \%$, comfortably surpassing the next runner up by $1.5 \%$ and trailing the second place model by only $0.26 \%$.
\end{abstract}

\section{Introduction}

The phenomenal growth of social media, web forums, and online reviews has spurred a growing interest in automated analysis of usergenerated text. User-generated text presents significant computational challenges because it is often highly disfluent. To address these challenges, we have begun to see a growing demand for tools and techniques to transform noisy usergenerated text into a canonical form, most recently in the Workshop on Noisy User Text at the Association for Computational Linguistics. This work describes a submission to the Lexical Normalization for English Tweets challenge as part of this workshop (Baldwin et al., 2015)
Motivated by the success of prior deep neural network architectures, particularly denoising autoencoders, we have developed an approach to transform noisy user-generated text into a canonical form with a feed-forward neural network augmented with a projection layer (Collobert et al., 2011; Kalchbrenner, Grefenstette, \& Blunsom, 2014; Vincent, Larochelle, Bengio, \& Manzagol, 2008). The model performs a character-level analysis on each word of the input. The absence of hand-engineered features and the avoidance of direct and indirect external data make this model unique among the three topperforming models in the constrained task.

This paper is organized as follows. In Section 2 we describe each component of our model. In Section 3 we describe the specific instantiation of our model, and in Section 4 we present and discuss results.

\section{Architecture and Components}

Our model consists of three components: a Normalizer that encodes the input and then reconstructs it in normalized form, a Flagger that determines whether the Normalizer should be used or if the word should be taken as-is, and a Conformer that attempts to smooth out simple errors introduced by quirks in the Normalizer.

In this section we will use the simple example transformation of " $u$ " to "you" where " $u$ " is the input text and "you" is the gold standard normalization. In our example we use a maximum word size of three. Figure 1 shows the flow of our example through the model. In broad overview, the input is preprocessed and sent to both the Nor- 
malizer and the Flagger. The Normalizer computes a candidate normalization, and the Flagger determines whether to use that candidate or the original word. The Normalizer's output is passed to the Conformer, which conforms it to a word in the vocabulary list, and then the candidate, the flag, and the original input word are passed to a simple decision component that either keeps the original word or uses the normalized version based on the output of the Flagger. While it may seem inefficient that the normalized version is always computed, even if it is not used, this approach is used so that the Normalizer and Flagger can be run in parallel on many inputs at once.

\subsection{Deep Feed-Forward Neural Networks}

As the central element of the Flagger and the Normalizer, the deep feed-forward neural network forms the basis of our model. A deep feedforward neural network takes a vector of numbers as input. This vector is known as a layer and each value within it is a neuron. The network
A deep feed-forward neural network can contain any number of hidden layers, each going through the same process, multiplying by a matrix of weights and transforming via a non-linearity. Hidden layers may also be of any size. Multiple applications of learnable weight matrices and non-linear transformations together allow a deep neural network to represent complex relationships between input and output (Bengio, 2009).

Deep feed-forward neural networks are trained by backpropagation. Backpropagation is a training method by which the gradient of any given weight in a network can be calculated from the error between the output of the network and a gold standard. It is described in more detail in (Rumelhart, Hinton, \& Williams, 1986).

\subsection{The Normalizer}

Our use of deep feed-forward neural networks for the task of normalization is inspired by the success of denoising autoencoders. (Vincent et al., 2008). Denoising autoencoders are neural

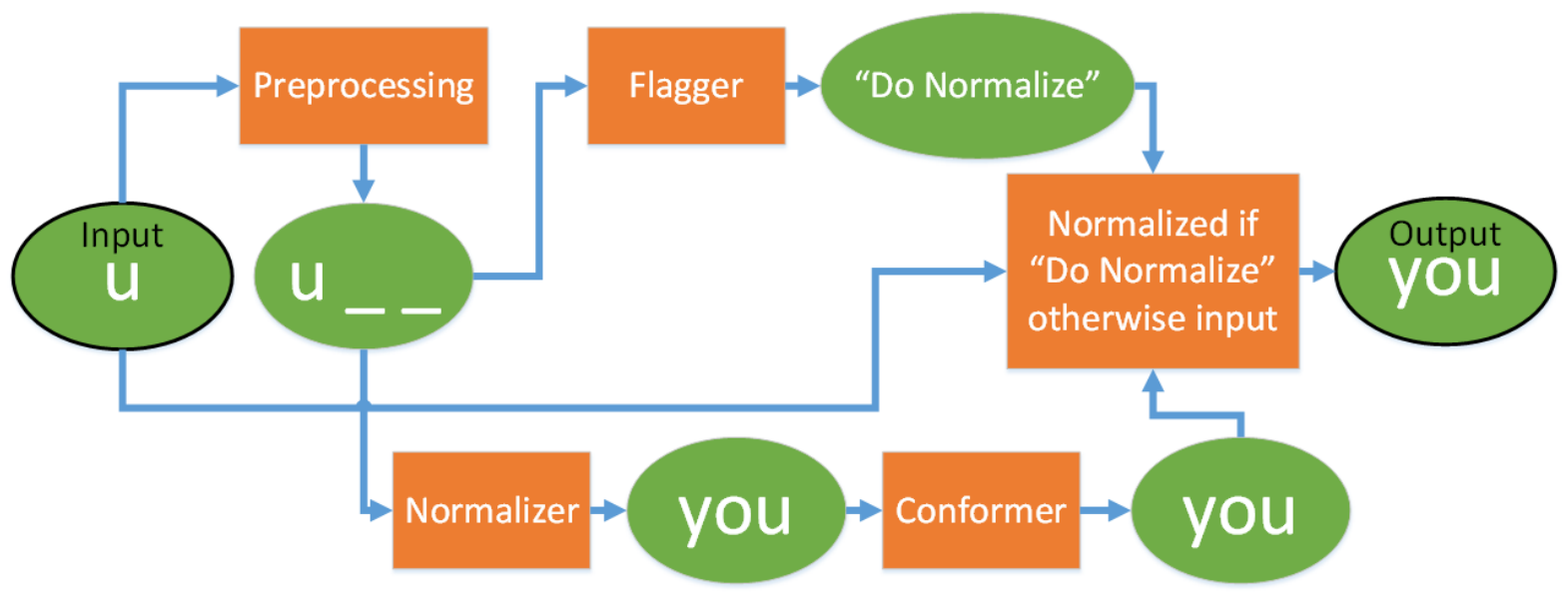

Figure 1: A flowchart detailing the process of normalizing a word. Information flows from left to right and ellipses represent data objects while rectangles represent processes.

multiplies the input layer by a matrix of weights to return another vector. This new vector is then transformed by a non-linearity. A number of functions can serve as the non-linearity, including the sigmoid and the hyperbolic tangent, but our model uses a rectified linear unit, given by the following expression.

$$
y=\max (x, 0)
$$

The rectified linear unit has been successful in a number of natural language tasks such as speech processing (Zeiler et al., 2013), and it was effective in an unpublished part-of-speech tagging model we developed.

The transformed vector is referred to as a hidden layer because its values are never directly observed in the normal functioning of the model. networks whose output is the same as their input. That is, they specialize in developing a robust encoding of an input such that the input can be reconstructed from the encoding alone. The denoising aspect refers to the fact that to encourage robustness, denoising autoencoders are given inputs that have been deliberately corrupted, or "noised" and are expected to reconstruct them without the noise. It is this "denoising" aspect that makes denoising autoencoders so interesting for text normalization.

The main component of our model, the Normalizer, uses a feed-forward neural network that functions on a similar principle to that of a denoising autoencoder. It reads the character sequence that describes the word and encodes it 
internally, outputting the denoised (normalized) version. It accomplishes this in three sets of layers. First the character projection layer takes a string and represents it as a fixed-length numeric vector. Next, a feed-forward neural network converts the data into its internal representation and, with a special output layer, into a denoised version of the input. Figure 2 shows a diagram of the Normalizer's architecture.

The first step of the Normalizer is performed by the character projection layer (Collobert et al., 2011). The character projection layer learns floating point vector representations of characters, which it concatenates into one large floating point vector word representation. In our example, the letter " $u$ " is represented by $n$ floating point numbers. For example, if $n=3$ the representation for " $u$ " might be $[0.1,-1.2,-0.3]$. This vector was chosen arbitrarily, but in the actual model, values are learned in training. The representations allow more information to be associated with a character than a simple numeric index.

In this simple example, the word " $\mathrm{u}$ " is composed of one character, but if it were longer, each letter would be separately represented. A key challenge at this point is that a feed-forward neural network cannot handle an arbitrary number of inputs. Because each position in the vector is a neuron matched directly to a set of weights, changing the size of the vector would require changing the size of the learned weights, and the model would have to be retrained.

To accommodate this, we use a fixed window. Before we send our input to the Normalizer, we comes $[\mathrm{u}, \ldots, \ldots]$ and then is projected and concatenated and becomes something like [0.1, -1.2, $0.3,1.3,0.0,-1.1,1.3,0.0,-1.1]$. Notice that we have nine values now in our input. That is the three values from " $u$ " and then the three values for " " " $([1.3,0.0,-1.1])$ twice, once for each " ". After this step, the system has a numeric vector representation of a word that is always the same length. It now sends it to the first layer of the feed-forward neural network. We deliberately select a large enough window that only in a small minority of cases does a word have to be reduced to fit into the window.

The last hidden layer's values go through one final matrix multiplication to output a list of values $w v$ in size, where $w$ is the size of the window and $v$ is the number of possible characters including the padding character, that is, the number of characters in the alphabet, which is shared between the input and output layers. In this last layer the nonlinear transformation is a special version of the softmax operation.

The softmax operation transforms a vector such that each of its values is between zero and one and the new vector sums to one. Mathematically, it is given as:

$$
\sigma(z)_{j}=\frac{e^{z j}}{\sum_{k=1}^{K} e^{z k}}
$$

Where $\mathrm{K}$ is the number of values in the vector. In our model, $\mathrm{K}=\mathrm{v}$, the size of the alphabet. These individual values can alternately be considered posterior probabilities for each of the possible decisions. If each value is mapped to a character,

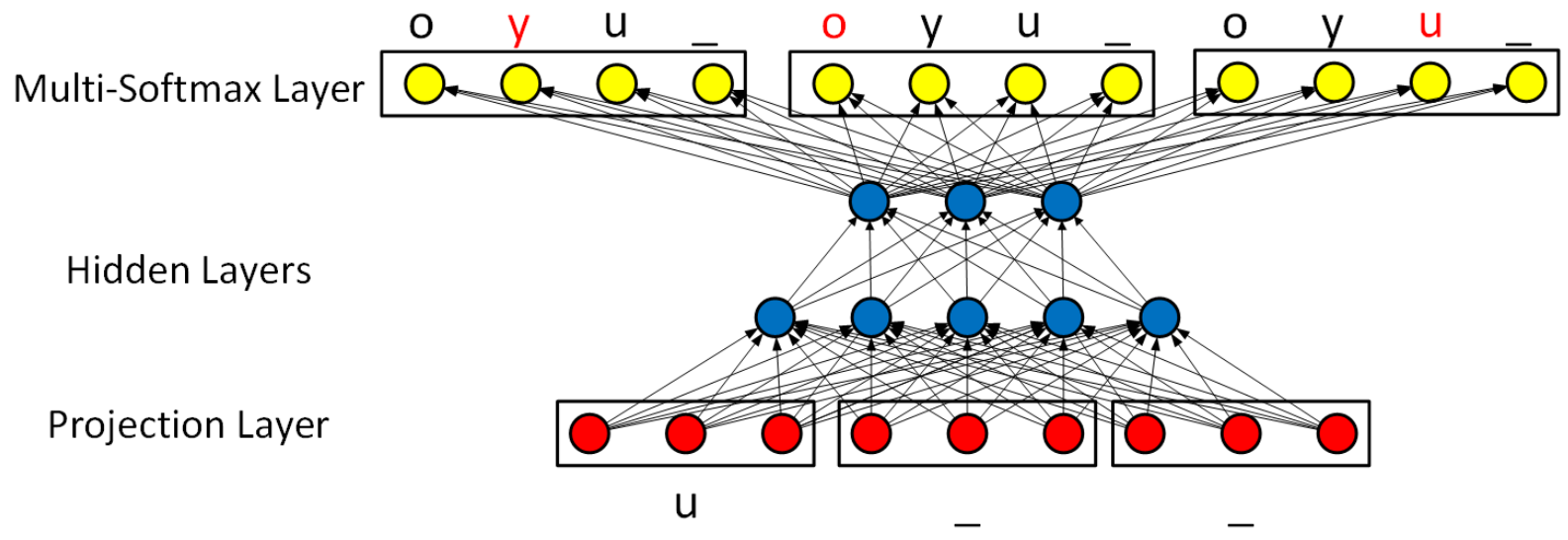

Figure 2: A diagram of the Normalizer correcting "u" to "you." The circles represent values, the lines weights.

preprocess it to meet a specified length, filling in unused spaces with a sentinel padding "character" that projects to its own set of learned weights like the other characters. Since the maximum word size in our example is 3 , we use a window of size 3 . Therefore, our input " $u$ " be- one can simply take the highest value to select the most likely character. In this case, we are predicting a window of $w$ characters rather than a single character, so we perform softmax separately on each of the $w$ sets of $v$ values in the layer. In prediction, we simply take the index of the 
highest value in each of the $w$ sets, but in training we take the whole prediction distribution and try to maximize the likelihood of each correct letter. We do not attempt to predict character embeddings because we are learning them, and the model would be likely to learn a trivial function with character embeddings that are all equal.

Training the Normalizer as a whole relies on generating posterior distributions and attempting to minimize the total negative log likelihood of the gold standard. Mathematically, our objective function is

$$
\cos \mathrm{t}=-\sum_{p \in P} \ln (p)
$$

Where $p$ is an element in $P$, the vector of the probabilities of each gold standard letter. So, if our model predicts " $y$ " as $75 \%$ likely for character 1 , "o" as $95 \%$ likely for character 2 , and "u" as $89 \%$ likely for character 3 in our window of size 3 , the negative log likelihoods calculated as $(.29, .05, .12)$ are summed to get the error. This sum error gives a simple measurement of performance to optimize, which backpropagates through the model to learn all the weights described above (Rumelhart et al., 1986).

\subsection{The Flagger}

The Flagger identifies what does and does not require normalization. The vast majority of the training data $(91 \%)$ does not require normalization, so returning the reconstructed encoding of every word would risk incorrectly regenerating an already canonical token.

The Flagger has the same general structure as the Normalizer itself except for the final layer. Instead of generating text at the last layer, a softmax layer predicts whether the token should be normalized at all. Thus, the Flagger's output layer is two neurons in size, one representing the flag "Do Normalize," and another representing the flag "Do Not Normalize." In the construction of the gold standard for the task, there were three reasons a token would not be normalized: firstly, the token is already correct, second, the token is in a protected category (hashtags or foreign words), or third, it was simply unrecognizable such that the human normalizer could not find the correct form. The Flagger accounts for but does not distinguish between these three possibilities.

\subsection{The Conformer}

Even when a token should be corrected, it is possible that the normalizer will come very close to correcting it without succeeding. Reconstructing the word "laughing," for instance, the normalizer can fail completely if it predicts even one letter wrong. An early analysis of validation data found that the normalizer had predicted "laugling" instead of laughing. These off-by-one errors are a frequent enough occurrence to merit a module to deal with them. The Conformer is also useful for correctly normalizing rare words whose correct normalization is too long for the window to represent. In particular "Imfao" expands to an impressive 27 characters, but if the Normalizer predicts only the first 25 characters, the Conformer can easily select the correct token.

To correct these small normalizer errors we construct the Conformer by collecting a dictionary from the gold standard training data. The dictionary is simply a list of all the unique words in the gold standard data. Then at runtime, whenever the Normalizer runs and predicts a word that is not present in the dictionary, we replace it with the closest word in the dictionary according to Levenshtein distance (Levenshtein, 1966). Ties are resolved based on which word comes first in the dictionary. Because Python's set function, which does not guarantee a specific order of its contents, is used to construct the dictionary, the dictionary's order is not predictable and thus ties are resolved unpredictably.

\section{Settings and Evaluation}

The model was implemented in Theano, a Python library for fast evaluation of multidimensional arrays using matrix operations (Bastien et al., 2012; Bergstra et al., 2010). We used Theano's implementation of backpropagation to train our model. For our window size, we selected 25 characters, which is large enough to completely represent $99.9 \%$ of the tokens in the training data while remaining computationally feasible. There are also a number of hyperparameters: the number and size of hidden layers, the size of character embeddings, and the dropout rate. We tried various combinations of values between 50 and 6000 for the size and 1 and 4 for the number of hidden layers in both our Normalizer and Flagger. Some combinations we tried can be seen in the results section. Especially large sizes and numbers of layers proved to require more memory than our GPU could support, and training them on our CPU was exceptionally slow. We also tried $50 \%$ and $75 \%$ dropout, meaning that during training we randomly excluded hidden nodes from consideration at each 
layer. Dropout has been shown to improve performance by discouraging overfitting on the training data, and $50 \%$ and $75 \%$ are common dropout rates (Hinton, 2014).

We found the highest F1 score on the validation data for the Normalizer with two hidden layers of size 2000 each and $50 \%$ dropout. This was close to the maximum size our GPU could support without reducing the batch size to be too small to take advantage of the parallelism. The Flagger's highest score was found at two hidden layers of size 1000 each and $75 \%$ dropout. Attempts to provide hidden layers of different sizes consistently found inferior results. For the size of each embedding in the character projection layer, 10 had proven effective earlier in a simpler unpublished Twitter part-of-speech task. We selected 25 for our character embedding size to account for the greater complexity of a normalization task.

We separated the provided training data into $90 \%$ training data, $5 \%$ validation data and $5 \%$ was held out as test data. In order to construct a useful model on the small amount of available data, we iterate training over the same data many times. Our model stopped training after 150 training iterations in which there was no improvement on the validation set. We chose 150 iterations as the smallest value that did not lead to ending the training at a clearly suboptimal value. The training also stops at 5,000 iterations but in practice it converged before reaching this value.

Early in development we found that the Normalizer had exceptional trouble reconstructing twitter-specific objects, that is, hash-tags (\#goodday), at-mentions (@marysue) and URLs (http://blahblah.com). Generally its behavior in all three cases was to follow the standard marker characters (@, \#, http://) with a string of gibberish unrelated to the word itself. Because these are protected categories that should not be changed, we removed them from the training data and rely on the Flagger to flag them as not to be corrected.

We used layer-wise pre-training, meaning we first trained with zero hidden layers (going directly from the character projection to the softmax layer) to initialize the character embeddings, then we trained with one hidden layer, initializing the character embeddings with their previously trained values. When we trained the full model using two hidden layers, we initialized both the character projection layer and the weights from the projected input to the first hid- den layer with the values learned before. The model continued to learn all the weights it used. Pretrained weights continued to be trained in the full model, although "freezing" some pretrained weights after pretraining and only training later weights in the full model has shown success when working with large amounts of unsupervised data and may be worthwhile to consider in future work (Yosinski, Clune, Bengio, \& Lipson, 2014).

Running on an NVIDIA GeForce GTX 680 GPU with 2 GB of onboard memory, training the Normalizer took about six hours. We do not include CPU and RAM specifications because they were not heavily utilized in the GPU implementation. The Flagger was considerably faster to train than the Normalizer, taking only a little over half an hour.

\section{Results and Discussion}

The model earned third place in the competition, with scores very close to the second place model. The model's results in the competition compared to the first, second, and fourth place models is shown in Table 1. The precision scores are much higher than the recall scores for all models because in this task precision measures the capability of the model to not normalize what does not need normalizing while recall requires that a model both correctly identify what needs to be normalized and correctly normalize it.

In addition to the challenge results, we performed a more in-depth analysis on our own held-out validation and test data. Our analysis of the scores is shown in Table 2.

Initial data on the Flagger is in Table 3. We further analyzed the different errors made on the validation data. Our findings can be found in Table 4. Given the large proportion of errors mistakenly marked "Do Not Normalize," we looked at these errors. A few examples can be found in Table 5. Although the Flagger was not trained with Normalizer confidence in mind, it does an impressive job of only cancelling a normalization when the normalization is either unnecessary or would fail. In no case did the Flagger prevent the Normalizer from making a correct normalization.

An analysis in Figure 3 shows some early results from using only the Normalizer without a Conformer or Flagger. To fit this many runs in a reasonable time span, we used only ten percent of the training data. In this analysis, error rate is measured by token. To put the error rates in perspective, our final error rate was close to three 
percent. We show this graph to illustrate a number of points. Particularly, we wish to illustrate the challenge of encoding and reconstructing every item in a massive vocabulary, the value of additional iterations of layer-wise pre-training, and the large spikes in the error rates at certain points in the model.

\begin{tabular}{|l|l|l|l|}
\hline \multicolumn{1}{|c|}{ Model } & \multicolumn{1}{c|}{ Precision } & Recall & F1-Score \\
\hline $\begin{array}{l}\text { NCSU_SAS } \\
\text { NING }\end{array}$ & 0.9061 & $\mathbf{0 . 7 8 6 5}$ & $\mathbf{0 . 8 4 2 1}$ \\
\hline $\begin{array}{l}\text { NCSU_SAS } \\
\text { WOOKHEE }\end{array}$ & $\mathbf{0 . 9 1 3 6}$ & 0.7398 & 0.8175 \\
\hline $\begin{array}{l}\text { NCSU_SAS } \\
\text { SAM }\end{array}$ & 0.9012 & 0.7437 & 0.8149 \\
\hline Iitp & 0.9026 & 0.7191 & 0.8005 \\
\hline
\end{tabular}

Table 1: Results of the constrained task

\begin{tabular}{|l|l|l|l|l|}
\hline \multicolumn{1}{|c}{ Data } & Precision & Recall & \multicolumn{1}{c|}{$\begin{array}{c}\text { F1- } \\
\text { Score }\end{array}$} & Accuracy \\
\hline $\begin{array}{l}\text { Valida- } \\
\text { tion }\end{array}$ & 0.8942 & 0.7752 & 0.8305 & 0.9740 \\
\hline Test & 0.8229 & 0.6870 & 0.7488 & 0.9656 \\
\hline
\end{tabular}

Table 2: Model Scores on Validation and Test Data

\begin{tabular}{|c|c|c|c|c|}
\hline Data & Precision & Recall & $\begin{array}{c}\text { F1- } \\
\text { Score }\end{array}$ & Accuracy \\
\hline $\begin{array}{l}\text { Valida- } \\
\text { tion }\end{array}$ & 0.9818 & 0.9939 & 0.9878 & 0.9776 \\
\hline Test & 0.9783 & 0.9930 & 0.9856 & 0.9736 \\
\hline
\end{tabular}

Table 3: Flagger scores on Validation and Test Data

\begin{tabular}{|l|c|}
\hline \multicolumn{1}{|c|}{ Error } & \multicolumn{1}{c|}{$\begin{array}{c}\text { Percentage } \\
\text { Occurrence }\end{array}$} \\
\hline $\begin{array}{l}\text { Correctly flagged, } \\
\text { misnormalized }\end{array}$ & $13.85 \%$ \\
\hline $\begin{array}{l}\text { Mistakenly flagged } \\
\text { "Do Not Normalize" }\end{array}$ & $66.15 \%$ \\
\hline $\begin{array}{l}\text { Mistakenly flagged } \\
\text { "Do Normalize" }\end{array}$ & $20.00 \%$ \\
\hline
\end{tabular}

Table 4: Analysis of errors. Percentages given are out of the total error count.

\begin{tabular}{|c|c|c|}
\hline \multicolumn{2}{|c|}{ Original } & \multicolumn{1}{c|}{$\begin{array}{c}\text { Gold Stand- } \\
\text { ard }\end{array}$} \\
\hline FB & Facebook & fabol \\
Fuhh & $\mathrm{f}^{* * *}$ & fuhh \\
OPENFOLLOW & open follow & openffolow \\
Feela & Feels & feela \\
Bkuz & because & bkuze \\
Kin & kind of & kin \\
Bruuh & brother & bruuhr \\
\hline
\end{tabular}

Table 5: Examples of tokens that were mistakenly flagged "Do Not Normalize." The "Normalized" column is what the model would have produced if the Flagger had produced the flag "Do Normalize"

The Normalizer demands much more representational power when not assisted by the Flagger. Before we added the Flagger, we saw continual improvement of results going up to four layers of six thousand nodes each. We saw greater improvements from adding more nodes per layer than from adding more layers. The cluster of three lines near the top all have layers of 1500 or 2000 nodes each, and the next cluster down is the models we tried with 4500 and 6000 nodes. Incidentally, all but the smallest of these models were too large for our GPU's 2GB of onboard memory. As a reminder, after we added the flagger, we only required two layers of 2000 nodes each to get competitive results. In each case we used a dropout rate of $50 \%$.

The default models pre-trained each layer for 250 iterations and we also trained models with the same structure for 500 iterations. We find a noticeable improvement in the error rate for the models that were pre-trained for more iterations. In the graph, the models with more pre-training make up the cluster of lines near the bottom of the graph.

Looking at the graphs, one may notice that some lines have brief spikes multiple percentage points in size. Because it only takes a one-letter mistake for a word to be misnormalized, we expect that at these times a small error arose that affected a large number of words. It is worth pointing out that each model continues to improve while in its spike, eventually dropping back to pre-spike levels.

The model is unique among the three topperforming models in that it avoids external data both directly and through indirect sources. The constrained task does not allow external data, but it does allow the use of off-the-shelf tools trained on external data. Our model does not use any such tools. Without the assistance of tools such as part-of-speech taggers, attempts to use context proved ineffective, likely because of increased sparsity. A given word that appears in the training set three hundred times may only appear three times after another particular word, and may not occur more than once with a particular prior word and following word, so it is more difficult to find patterns in limited data. Future work could either attempt to use tools to provide additional information or could simply take advantage of large amounts of data to learn directly the relationships such tools traditionally abstract for the benefit of conventional machine learning.

There is one other point: the human graders often made different decisions about whether or what a term should be normalized to. For exam- 
ple, sometimes the word "pics" used to refer to pictures was normalized to "pictures" but other times it was left as "pics". These inconsistencies in the gold standard make it difficult to accurately judge the quality of the models submitted. Occasionally when we examined mistakes the model made, we found that the model's prediction was correct according to the gold standard, but that the gold standard was wrong. An inter-rater reliability measure would help us to gauge not only how well our models compare to each other but how they compare to agreement between human coders. this happened much less often than having the system normalize incorrectly. A model that predicts words from a vocabulary instead of reconstructing them would be faster to train and would not require a Conformer, and, considering the top two models were vocabulary based, might outperform our reconstruction-based model.

A second direction for future work centers on leveraging external data. With more time and greater computing power, it may be the case that it is possible to learn sophisticated language models in an unsupervised fashion from both standard conversational text and twitter data.

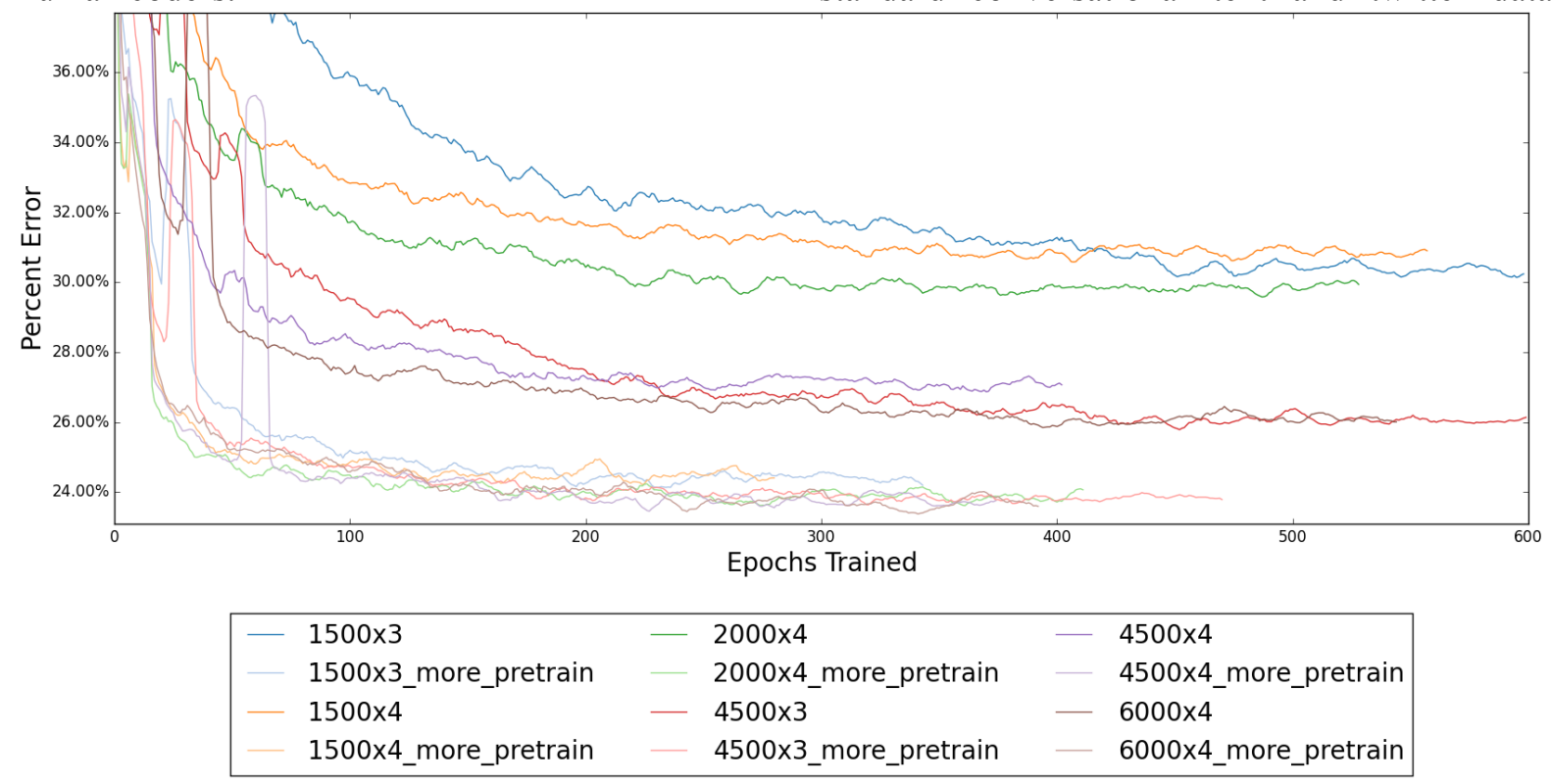

Figure 3: The Normalizer component validation scores by epoch. Model structures are given by "LxN" where L is the size of each layer and $\mathrm{N}$ is the number of layers and more_pretrain indicates that pretraining has continued for 500 instead of 250 iterations, and they cluster at the bottom with the lowest error. To smooth the graphs and make them more interpretable, values at each epoch are the average of a 10 -epoch window.

\section{Conclusions and Future Work}

Normalization of Twitter text is a challenging task. With a direct application of simple deep learning techniques and without relying on any sources of external data, direct or indirect, we built a model that performed competitively with the other models in the task. Our method shows the ability of deep learning to tackle complex tasks without labor-intensive hand-engineering of features.

An important direction for future work is simplifying the normalization pipeline. The need for a Conformer in particular suggests that there is room for improvement in the model. Although constructing the normalized form rather than selecting from a list leaves the possibility open that a system could normalize to a correct word that did not appear in the training data, in practice
With this additional data, a model may be able to effectively use context in distinguishing between multiple possible normalizations of a word. Denoising autoencoders in particular are known to make good use of unsupervised data.

A third direction for future work is to investigate more challenging normalization tasks that include correction of syntax and do not present the text already tokenized. These will give us an opportunity to attempt tasks closer to the challenges our normalization systems will face in the real world.

Finally, it will be important to investigate the overall utility of normalization of text as a preprocessing step for other analysis. While many tasks will only benefit from cleaning the data, it is not clear that the canonical forms of words retain the same connotations that the original "noisy" versions held. For a simple example, if we were to normalize "cooooooool" to "cool" we 
would lose the emphasis implied by the elongation of the vowel. For some tasks, it may be important to retain the information contained in such non-canonical forms.

\section{References}

Baldwin, Timothy, Catherine, Marie, Han, Bo, Kim, Young-Bum, Ritter, Alan, \& Xu, Wei. (2015). Shared Tasks of the 2015 Workshop on Noisy User-generated Text : Twitter Lexical Normalization and Named Entity Recognition. In Proceedings of the Workshop on Noisy Usergenerated Text (WNUT 2015). Beijing, China.

Bastien, Frédéric, Lamblin, Pascal, Pascanu, Razvan, Bergstra, James, Goodfellow, Ian, Bergeron, Arnaud, ... Bengio, Yoshua. (2012). Theano: New Features and Speed Improvements. In Deep Learning and Unsupervised Feature Learning NIPS 2012 Workshop (pp. 1-10). Retrieved from http://arxiv.org/abs/1211.5590v1

Bengio, Yoshua. (2009). Learning Deep Architectures for AI. Foundations and Trends ${ }^{\circledR}$ in Machine Learning, 2(1), 1-127. http://doi.org/10.1561/2200000006

Bergstra, James, Breuleux, Olivier, Bastien, Frédéric, Lamblin, Pascal, Pascanu, Razvan, Desjardins, Guillaume, ... Bengio, Yoshua. (2010). Theano: A CPU and GPU Math Compiler in Python. In Proceedings of the 9th Python in Science Conference (pp. 3-10). Austin, Texas.

Collobert, Ronan, Weston, Jason, Bottou, Leon, Karlen, Michael, Kavukcuoglu, Koray, \& Kuksa, Pavel. (2011). Natural Language Processing (almost) from Scratch. The Journal of Machine Learning Research, 12, 2493-2537. Retrieved from http://dl.acm.org/citation.cfm?id=2078186

Hinton, Geoffrey. (2014). Dropout: A Simple Way to Prevent Neural Networks from Overfitting. The Journal of Machine Learning Research, 15, 19291958.

Kalchbrenner, Nal, Grefenstette, Edward, \& Blunsom, Phil. (2014). A Convolutional Neural Network for Modelling Sentences. ACL, 655-665.

Levenshtein, Vladimir. (1966). Binary Codes Capable of Correcting Deletions, Insertions, and Reversals. Soviet Physics Doklady, 10(8), 707-710.

Rumelhart, David, Hinton, Geoffrey, \& Williams, Ronald. (1986). Learning Representations by Back-propagating Errors. Nature, 323(9), 533-
536. Retrieved from http://books.google.com/books?hl=en \&lr=\&id=FJ blV_iOPjIC\&oi=fnd\&pg $=$ PA2 $13 \& d q=$ learning + re presentations + by+backpropagating+errors\&ots $=\mathrm{zZEk} 5 \mathrm{hHYWU} \&$ sig $=\mathrm{B} 8$ 6wdYsAvCWVEN3aA-RCmw8_IJ8

Vincent, Pascal, Larochelle, Hugo, Bengio, Yoshua, \& Manzagol, Pierre-antoine. (2008). Extracting and Composing Robust Features with Denoising Autoencoders. Proceedings of the 25th International Conference on Machine Learning ICML '08, (July), 1096-1103. http://doi.org/10.1145/1390156.1390294

Yosinski, Jason, Clune, Jeff, Bengio, Yoshua, \& Lipson, Hod. (2014). How Transferable are Features in Deep Neural Networks? In Advances in Neural Information Processing Systems 27 (pp. $1-9)$.

Zeiler, M. D., Ranzato, M., Monga, R., Mao, M., Yang, K., Le, Q. V., ... Hinton, G. E. (2013). On Rectified Linear Units for Speech Processing. ICASSP, IEEE International Conference on Acoustics, Speech and Signal Processing Proceedings, 3517-3521. http://doi.org/10.1109/ICASSP.2013.6638312 